\title{
The structure of species discrimination signals across a primate radiation
}

Sandra Winters ${ }^{1}$, William L. Allen ${ }^{1,2}$, \& James P. Higham ${ }^{1}$

$4{ }^{1}$ Department of Anthropology, New York University, 25 Waverly Place, New York, NY 10003,

5 USA

$6 \quad{ }^{2}$ Department of Biosciences, Swansea University, Wallace Building, Singleton Park, Swansea

7 SA2 8PP, Wales, UK

9 Discriminating between conspecifics and heterospecifics potentially challenging for closely

10 related sympatric species. The guenons, a recent primate radiation, exhibit high degrees of

11 sympatry and form multi-species groups in which hybridization is possible but rare in most

12 populations. Guenons have species-specific colorful face patterns hypothesized to function in

13 species discrimination. Here, we apply a novel machine learning approach to identify the face

14 regions most essential for correct species classification across fifteen guenon species. We then

15 demonstrate the validity of these computational results using experiments with live guenons,

16 showing that facial traits identified as critical for accurate classification do indeed influence

17 selective attention toward con- and heterospecific faces. Our results suggest variability among

18 guenon species in reliance on single-trait-based versus holistic facial characteristics when

19 discriminating between species, and differences in behavioral responses to faces can be linked to

20 whether discrimination is based on a single trait or whole-face pattern. Our study supports the

21 hypothesis that guenon face patterns function to promote species discrimination and provides

22 novel insights into the relationship between species interactions and phenotypic diversity.

23 Keywords: species discrimination, visual signal form, complex signals, guenons 


\section{Introduction}

26 Closely related species living in sympatry face a potential challenge in discriminating between

27 conspecifics and heterospecifics. Such decision-making has important selective outcomes,

28 particularly in behaviors such as mate choice, with individuals choosing heterospecific mates

29 often incurring substantial fitness costs [1]. One mechanism for avoiding the costs if interacting

30 with heterospecifics is the use of species-specific signals that structure behavioral interactions

31 between species. For instance, mating signals and associated mating preferences that differ

32 between sympatric heterospecifics can function to maintain reproductive isolation across species

33 boundaries [2]. Such signals are predicted to be salient and distinctive [3], with sympatric species

34 under selective pressure to diversify. A pattern in which signal distinctiveness increases with

35 degree of sympatry, known as character displacement [4,5], has been observed in a wide variety

36 of animal groups [6-13]. Importantly, signals that function to maintain reproductive isolation via

37 mate choice should elicit increased mating interest from conspecifics compared to

38 heterospecifics [14].

39 Species in evolutionarily young animal radiations may be at particular risk of

40 hybridization and other costly interactions with heterospecifics due to behavioral similarities and

41 a lack of post-mating barriers to reproduction [15]. One such radiation is the guenons (tribe

42 Cercopithecini), a group of African primates consisting of 25-38 recognized species [16-18] that

43 diverged from papionin primates around 11.5 million years ago [19]. Guenons exhibit high

44 degrees of sympatry and often form polyspecific groups in which multiple species travel and

45 forage together [20]. Many guenons therefore interact with heterospecifics that share general

46 patterns of morphology (e.g. overall body size/shape) and behavior (e.g. activity patterns). In 
47 such circumstances, discriminating between con- and heterospecifics may be particularly

48 important, especially in a mating context. Hybridization between sympatric guenon species is

49 possible but rare in natural circumstances [21], suggesting the existence of barriers to

50 heterospecific mating within mixed-species groups.

51 Guenons are among the most colorful and visually patterned groups of primates with

52 many species exhibiting extraordinary and unique face markings [10,23,25-27], which are

53 minimally variable between sexes across all guenon species [23,24]. Kingdon [23,26,27]

54 hypothesized that guenons use their divergent facial appearances to distinguish between species

55 and therefore select appropriate mates. This young and impressively diverse primate radiation

56 represents a fascinating test case of how visual signals are involved in species radiations and

57 mixed-species interactions [5,28-30]. Recent empirical work has begun to generate evidence for

58 their key role in guenon phenotypic and species diversification. Images of guenon faces can be

59 reliably classified by species using computer algorithms [10,24], demonstrating that guenon

60 faces contain species-specific identifying information. Guenon face patterns also exhibit

61 character displacement, with facial distinctiveness between species increasing with degree of

62 sympatry across the group [10]. Moreover, facial components common across species (nose

63 spots and eyebrow patches) alone can be used to computationally classify species [24]. This

64 suggests that guenon faces may be somewhat modular, with species information encoded in

65 particular face regions. Which face regions are most important, and the extent to which such

66 regions vary across species remains an open question that is of key importance to understanding

67 how complex signals involved in species discrimination evolve. Critically, it is unknown whether

68 variation across guenon species in purported species discrimination signals is perceived and

69 acted on by con- and heterospecific receivers. 
Here, we use a machine learning approach to identify guenon face regions that are most

71 important for correct species classification by a computer. These results objectively identify the

72 signal components most likely to be useful to guenon receivers. We use them to determine which

73 signal properties to systematically investigate in behavioral experiments with guenon observers.

74 The machine-learning stage is critical, as many experiments that investigate behavioral responses

75 to complex signals select manipulations based on the perceptions of investigators, which

76 introduces anthropocentric bias [31]. Using the guenon face image database produced by Allen et

77 al. [10], we couple eigenface decomposition of the faces [32] with a novel occlude-reclassify

78 scheme in which we systematically block each part of the face and reclassify the image. This

79 allows us to document the spatial distribution of species-typical information across guenon faces

80 by identifying which face regions, when obscured, cause the break-down of correct species

81 classification. Eigenface decomposition was originally developed for individual face

82 discrimination in humans [32]; feature detection based on eigenfaces is also applicable to other

83 types of discrimination tasks involving complex animal signals [33-35] and has been used

84 previously to quantify guenon facial variation [10]. The perceptual face space generated by

85 eigenface decomposition parallels mammalian visual processing [36], lending biological

86 credibility.

87 After identifying the face regions that cause break-down in classification, and thus those

88 that should be important for correct species identification, we then present captive putty nosed

89 monkeys (Cercopithecus nictitans) and mona monkeys (C. mona) with images of con- and

90 heterospecific faces exhibiting variation in these regions and measure their resulting eye gaze to

91 assess their ability to distinguish between species based on face patterns. Ours is the first direct

92 measure of guenon responses to con- and heterospecific faces, which is crucial for clarifying the 
93 biological relevance of guenon face patterns and for validating previous correlational results.

94 Differences in looking time between classes of stimuli can be difficult to interpret due to various

95 and often unpredictable novelty and familiarity effects [37], however primates reliably exhibit a

96 visual bias (i.e. greater looking time) toward images of conspecifics compared to those of

97 heterospecifics [38-42]. We follow the interpretation that longer looking time at a particular face

98 reflects level of interest. This is consistent with an interpretation that the face resembles a

99 conspecific face more closely, though other explanations are possible.

Our experimental trials involve the simultaneous presentation of paired con- and

101 heterospecific faces, focusing on a particular facial trait for each species. For putty nosed

102 monkeys we focus on nose spots and for mona monkeys on eyebrow patches, on the basis that

103 each of these features is within the region of the face identified by our machine learning

104 approach as being critical for that species. In each trial, heterospecific faces either do or do not

105 share a focal face trait with the subject, and conspecific faces are presented either naturally or

106 after being modified to remove the focal trait (for example stimuli, see Figure 1). This approach

107 allows us to assess generalized species biases in degree of interest as well as the extent to which

108 particular face regions influence these biases.

We predicted variability across species in the face regions identified by our occlude-

110 reclassify procedure, but made no predictions regarding which regions in particular would be

111 essential for each species. In looking time experiments, we predicted that putty nosed and mona

112 monkeys would exhibit visual biases toward face images of conspecifics, and that these biases

113 would be influenced by species-typical facial characteristics identified as important for correct

114 species classification. Such a pattern of results would support a role for species discrimination

115 signals likely used to facilitate inter-specific interactions such as maintaining reproductive 
116 isolation via mate choice in generating and maintaining phenotypic variation in one of the most

117 speciose and diverse primate radiations. Species discrimination requires differentiating between

118 conspecifics and all heterospecifics at the very least, however it may be possible that animals

119 may also be able to discriminate between different heterospecific species. Ultimately, by

120 examining how aspects of highly complex signals encode species identity and influence receiver

121 biases, this research increases our understanding of how selection for species identity signaling

122 generates phenotypic diversity.

123

124 Methods

125 Image collection \& processing

126 Guenon face pattern analyses are based on an existing database of guenon face images from 22

127 guenon species [10]. Detailed methods of image collection and processing have been published

128 elsewhere [10]. Briefly, we used digital images of captive guenons collected using a color-

129 calibrated camera. Multiple images were taken of each subject while in a front-facing position

130 under indirect light. Images were transformed from camera RGB color space to guenon LMS

131 color space, defined by the peak spectral sensitivities of guenon long, medium, and short

132 wavelength photoreceptors. All images were then standardized with respect to illumination, size,

133 blur, and background. Each image was resized to be 392 by 297 by 3 pixels. All pixel values

134 were represented using double-level precision.

135 To avoid classifying species based on a very small number of exemplars, we restricted

136 our analyses to species represented by at least four individuals in our image database (i.e. all

137 classifications in a leave-one-out procedure are made based on at least three exemplars; see 
138 below). Our analysis is therefore based on 599 total images of 133 individuals, collectively

139 representing fifteen guenon species (for species-specific sample sizes, see Figure 3).

141 Identification of face regions important for species classification

142 Guenon face images can be reliably classified by species based on eigenface features [10,24].

143 This approach relies on dimensionality reduction via principal component analysis (PCA) to

144 extract relevant features from face images; these features can then be used for the classification

145 of new faces [32]. In this procedure, each 'eigenface' (i.e. the eigenvectors resulting from PCA

146 of all face images) represents a different dimension of facial variability and each face image can

147 be represented by a series of weights associated with each eigenface. This creates a multi-

148 dimensional 'face space' in which faces are represented as points based on their eigenface

149 weights, and zero weights for all eigenfaces (i.e. the center of the space) represents the average

150 face across all images. Such face spaces have psychophysical parallels in primate face processing

151 centers in the visual cortex [36]. Multiple images of each subject were averaged to generate

152 average individual faces, which in turn were used to generate the average species faces that were

153 used in eigenface decomposition. We classified new images using a nearest-neighbor classifier

154 based on minimum Euclidean distance to each average species face in face space. This scheme

155 corresponds to an average face model of guenon face learning, which assumes that guenons

156 cognitively encode different species' face patterns as the mean of all encountered examples. In

157 previous work using similar methods, results were robust to the choice of learning model [10].

158 To avoid using the same individual guenons to both train and test our species classifier

159 we used a leave-one-out procedure for all analyses. For this procedure, we systematically

160 removed each individual from the image set, repeated the analysis procedure outlined above, 
161 then classified each image of the excluded individual based on the features generated from all

162 other images. All species included in these analyses are represented by at least four individuals

163 (range: 4-23). We present results for all species, however results for species with samples sizes

164 in the lower end of this range should be considered less robust and interpreted with caution.

165 Eigenface-based features can be used to reliably classify guenons by species based on

166 axes of variation, however the extent to which specific facial characteristics are relevant for

167 correct classification of each species is difficult to determine. We used an occlude-reclassify

168 scheme developed to identify which image regions contribute most to correct classification in

169 computer vision classification tasks [43]. For each correctly classified image, we systematically

170 blocked each image region and re-classified the image; a correct re-classification indicates that

171 the occluded region of the face was unnecessary for correct classification, while an incorrect re-

172 classification indicates that the occluded region was essential. Occlusion of face regions was

173 accomplished by setting the relevant pixel as well as all those in a thirty-pixel radius to the mean

174 face color of that species. This procedure was repeated for every pixel in the image, effectively

175 sliding the occluded region across all face areas. A radius of thirty pixels occludes approximately

176 five percent of the image (Figure 2), with the specific region being occluded shifting by one

177 pixel at each iteration. Primate faces are broadly symmetrical, therefore to avoid the presence of

178 duplicate spatial information that may support species classification when part of the face is

179 occluded, we ran analyses on the left and right halves of the face separately. Results differed

180 little, so for clarity we report the results from the left hemi-face classification in the main text,

181 with right-side results summarized in the supplementary results. For more details on the

182 implementation of the occlude-reclassify procedure, see supplementary methods. Based on this

183 occlude-reclassify scheme, we generated a binary image for each image in our data set, with each 
184 pixel being either zero (black) or one (white) base on whether the image was correctly classified

185 when that pixel and its neighbors was occluded. We then averaged these binary images across

186 individuals and species to generate species level heatmaps depicting face regions that are

187 essential for correct classification across species. For visualization, we converted greyscale

188 heatmaps to color using a color mapping function. To facilitate the identification of critical face

189 regions, occlusion results are presented as composite images combining heatmaps and a

190 greyscale version of the relevant species average face, with transparency set to 0.5 .

191 Heatmaps vary across species in the extent to which face regions identified as essential

192 for correct species classification are spread across the face (i.e. ranging from small and isolated

193 face regions to large portions of the face identified as critical) as well as the relative import of

194 identified regions (i.e. the likelihood that identified regions caused misclassification, encoded as

195 how dark identified regions are in the heatmap). To quantify the spread and relative importance

196 of the identified face regions across species, we calculated the proportion of the face

197 misclassified and the mean classification error, respectively. The proportion of the face

198 misclassified was calculated as the number of heatmap pixels less than one (i.e. those that were

199 ever incorrectly classified) divided by the total number of pixels in the average face for each

200 species; higher values indicate that the face regions essential for correct species classification are

201 spread more widely across the face. The mean classification error was calculated as the mean

202 value of all heatmap pixels less than one; higher values indicate that the face regions identified

203 are particularly critical and more often lead to misclassification when occluded (i.e. the identified

204 regions are darker in the heatmaps). Computational analyses were conducted in MATLAB ${ }^{\mathrm{TM}}$ and

205 run on the High Performance Computing cluster at New York University. 
208 Looking time experiments were conducted at CERCOPAN sanctuary in Calabar, Nigeria, and

209 included 18 adult putty nosed monkeys (6 males, 12 females) and 16 adult mona monkeys (10

210 males, 6 females). Each species was divided into four experimental groups (based on socially

211 housed groups), with all individuals in the group viewing the same images in the same order. In

212 each species, two experimental groups were presented with male stimulus images and two with

213 female stimulus images across all trials. Experiments involved the simultaneous presentation of

214 two stimulus images to subjects, with their resulting eye gaze measured to determine visual

215 biases. Stimulus preparation and experimental procedures were carried out following the

216 recommendations of Winters et al. [37]. Briefly, we prepared stimulus images depicting guenon

217 faces which were presented approximately life-sized (image size on screen: $500 \times 500$ pixels,

$21811.96 \times 11.96 \mathrm{~cm})$, with accurate colors, and standardized for relevant characteristics. Stimulus

219 image pairs were presented to subjects side-by-side using a custom-designed experimental

220 apparatus. For more details regarding subjects, stimuli preparation, and experimental apparatus

221 design, see supplemental methods.

222 Each subject participated in three trials, with stimulus image pairs depicting the

223 following: (1) a conspecific and a heterospecific that shares a focal trait with the conspecific, (2)

224 a conspecific and a heterospecific that does not share a focal trait with the conspecific, and (3) a

225 conspecific for which the focal trait has been modified and a heterospecific that shares the focal

226 trait with the conspecific. Heterospecifics presented to putty nosed monkeys were Wolf's

227 guenons (C. wolfi, no nose spot) and red-tailed monkeys (C. ascanius, nose spot); heterospecifics

228 presented to mona monkeys were red-tailed monkeys (no eyebrow patches) and Diana monkeys

229 (C. diana, eyebrow patches). Heterospecific species were selected based on the presence/absence 
230 of the relevant facial trait, a lack of range overlap with the subject species, and availability of

231 sufficient and appropriate images in our database. Image presentation locations (i.e. left verses

232 right) were counterbalanced across trials, and trial order was varied across subjects; both factors

233 were included in statistical analyses. For each trial, we placed the experimental apparatus

234 immediately outside the relevant enclosure and recorded the identities of participating subjects.

235 We waited a minimum of one week between trials of the same subject to minimize habituation or 236 trial order effects.

Videos of each trial were coded frame by frame to quantify the amount of time subjects

238 spent looking at each stimulus image. All coding was done blind to trial conditions and stimulus

239 image location. Reliability was assessed using approximately $10 \%$ of all trial videos, in which

240 we assessed agreement between two coders on the direction of jointly coded looks within these

241 trials as being in agreement in $94.46 \%$ of frames (Cohen's kappa $=0.883$ ), which is well within

242 the range of acceptable reliability scores for this type of data [37,44]. Raw looking time data was

243 compiled to yield a total number of frames spent looking at each stimulus image for each subject

244 in each trial. Subjects varied widely in their level of interest in experiments, resulting in

245 considerable variation in overall looking time. We therefore used only the first five seconds of

246 looking for each subject in each trial, while allowing them to complete the current look at the

247 five second mark (i.e. we required at least one second of non-looking before terminating coding

248 for each subject). This resulted in a mean total looking time ( \pm standard deviation) of $3.89 \mathrm{~s}( \pm$

$2491.98 \mathrm{~s})$ for putty nosed monkeys and 4.58s $( \pm 2.52 \mathrm{~s})$ for mona monkeys, which is similar to

250 durations reported in previous looking time experiments in primates $[37,44]$. Because a direct

251 comparison is made between the species depicted in stimuli, each trial effectively serves as its

252 own control. 
254 Statistical analyses

255 We analyzed differences in looking time elicited by subjects in experimental trials using

256 generalized linear mixed models (GLMMs). Models were fit using a binomial family

257 distribution, with the number of video frames spent looking at the targeted stimulus image and

258 the number of video frames spent looking at the paired image set as the binomial outcome

259 variable. This structure allowed us to assess looking biases while accounting for any differences

260 in total looking time across subjects. All models included group, subject, and unique trial (i.e. a

261 unique identifier for each subject in each trial, included to account for our analysis of the two

262 images presented in each trial as separate data 'rows') as nested random effects. Stimulus species

263 (conspecific v. heterospecific) and focal trait similarity (presence of nose spots for putty nosed

264 monkeys and eyebrow patches for mona monkeys), were included as fixed effects. We also

265 included the following additional factors as fixed effects: subject age (log transformed), sex, and

266 origin (captive v. wild born); stimulus image presentation spot (right v. left), eye contact (direct

267 eye contact with the camera or looking slightly away), sex, and degree of familiarity to the

268 subject; and trial order, apparatus pattern, and display ICC profile. For more details about these

269 variables see supplemental methods.

270 To determine which variables significantly influenced subject looking biases, we

271 compared models with different parameterizations using likelihood ratio tests (LRTs). A single

272 model including all fixed effects simultaneously would involve an excessive number of

273 predictors. We therefore first analyzed each variable separately via comparisons to a null model

274 including only random effects, and excluded non-significant predictors from subsequent

275 analyses. We generated an initial model composed of factors that were statistically significant 
276 (alpha $<0.05)$ or exhibited a trend $($ alpha $<0.1)$ when tested alone. To determine the statistical

277 significance of these factors we then systematically excluded each factor from this model and

278 tested its contribution to the fit of the model to the data using LRTs. When species (conspecific

279 v. heterospecific) and focal trait (shared v. not shared) were both significant predictors in this

280 model we also tested a species*trait interaction. Within a final model composed of significant

281 predictors we compared across factor levels of fixed effects using z scores calculated using a

282 normal approximation. Adherence to model assumptions was verified based on plots of fitted

283 values and residuals. Trials from putty nosed and mona monkeys were analyzed separately.

284 GLMMs were run using the 'Ime4' package version 1.0.12 [45] in R version 3.3.3 [46].

\section{Results}

287 Occlude-reclassify machine classification

288 We began by confirming that guenons could be reliably classified by species based on eigenface 289 decomposition [10]. Average subject images were correctly classified by species $99.31 \%$ of the

290 time, and distinct images were correctly classified $93.03 \%$ of the time. All correctly classified

291 images $(n=654)$ were used to identify face regions of critical importance to correct species

292 classification by the computer algorithm, using our occlude-reclassify scheme. We identified

293 essential face regions in all guenon species that, when occluded, led to incorrect species

294 classification (Figure 3; for full resolution images see Supplementary File 1). Species differed in

295 the importance of different face regions as well as the extent to which important regions were 296 concentrated in specific facial features or were more widely distributed across larger face areas

297 (Figure 4). For example, the nose spot of the putty nosed monkey was the most critical facial 298 feature identified across all species. The putty nosed monkey had the highest mean error rate for 
misclassified face regions - indicating that the face regions identified had the highest likelihood

300 of causing misclassification when occluded - with the essential regions centered exclusively on

301 the nose. Thus, in the putty nosed monkey the nose is the only essential face feature; when the

302 nose is occluded species classification breaks down, whereas occluding any other face region has

303 no effect. In contrast, in other species our classifier relied on broader regions of the face, with

304 larger face regions identified as important for correct classification and the classifier relying less

305 exclusively on a single feature. The mona monkey is a good example of this, with disparate face

306 regions including the cheeks, eyebrows, and ear tufts all influencing correct classification of this

307 species. In some species negative space is important, suggesting that what makes the faces of

308 species distinctive may be the absence of certain facial traits. For instance, in M. talapoin the

309 absence of distinctive traits along the sides of the face - such as cheek and/or ear tufts observed

310 in other species - appears to be important.

Looking time experiments

313 Our experiments presenting subjects with pairs of con- and heterospecific faces revealed visual

314 biases in resulting eye gaze in both putty nosed and mona monkeys. In the subset of trials that

315 included a natural conspecific and a heterospecific without the relevant face trait (i.e. those

316 where the relevant facial traits are not spread across both con- and heterospecific faces), species

317 (and therefore also facial trait) was a significant predictor of looking behavior (putty nosed

318 monkeys: Chisq $=63.312, \mathrm{p}<0.001$; mona monkeys: Chisq $=30.755, \mathrm{p}<0.001$, with both

319 putty nosed and mona monkeys exhibiting a conspecific bias (respectively: $\mathrm{z}=7.920, \mathrm{p}<0.001$;

$320 \mathrm{z}=5.536, \mathrm{p}<0.001 ;$ Figure 5). 
Across all trials, in putty nosed monkeys model comparisons revealed that looking

322 behavior was significantly influenced by facial trait (nose spot v. no nose spot; Chisq $=11.511, \mathrm{p}$

$323<0.001$ ) and image location (right v. left; Chisq $=18.065, \mathrm{p}<0.001$ ), but not by species

324 (conspecific v. heterospecific; Chisq $=3.051, \mathrm{p}=0.081$ ). Overall, putty nosed monkeys looked

325 longer at stimulus faces that displayed a white nose patch $(\mathrm{z}=3.343, \mathrm{p}<0.001$; Figure 5), their

326 diagnostic species trait, regardless of species identity. Putty nosed monkeys also exhibited a

327 significant right gaze bias $(\mathrm{z}=4.289, \mathrm{p}<0.001)$. None of the other variables relating to subject,

328 stimulus, or trial characteristics were statistically significant (all $\mathrm{p}>0.1$; Supplementary Table

$3291)$.

In mona monkeys, model comparisons revealed that looking behavior was significantly

331 influenced by species (conspecific v. heterospecific; Chisq $=177.480, \mathrm{p}<0.001$ ), facial trait

332 (eyebrow patches v. no eyebrow patches; Chisq $=29.462, \mathrm{p}<0.001$ ) and a species*trait

333 interaction $($ Chisq $=8.242, \mathrm{p}=0.004)$. Across all trials, mona monkeys looked longer at

334 conspecifics $(\mathrm{z}=9.945, \mathrm{p}<0.001$; Figure 5) and as a separate effect, faces without white

335 eyebrow patches, one component of their overall wider diagnostic discrimination area $(\mathrm{z}=5.851$,

$336 \mathrm{p}<0.001)$. There was also an interaction between these two variables, with mona monkeys

337 looking longer at heterospecific faces with white eyebrow patches $(z=2.868, p=0.004)$. None

338 of the other variables relating to subject, stimulus, or trial characteristics played a significant role

339 in mona monkey visual biases (all $\mathrm{p}>0.1$; Supplementary Table 2).

\section{Discussion}

342 Our experiments show that eye gaze in guenons is influenced by face regions identified as

343 critical to correct species classification by our machine classifier. This convergence of results 
344 using disparate methods reinforces the validity of both, and ties computationally derived results

345 directly to guenon perception, demonstrating the utility of machine learning for identifying

346 biologically relevant signal components. To our knowledge, ours is the first analysis to use

347 machine classification combined with the systematic occlusion of image regions to characterize

348 the relevant signaling information encoded in an animal's appearance. This approach, based on

349 research in the field of computer vision designed to assess the contribution of image contents to

350 object classification [43], is useful for objectively quantifying the relative roles of different

351 signal components with respect to overall signal function. In closely-related sympatric species,

352 selection against mating or interacting with heterospecifics is often associated with the evolution

353 of species-typical traits used to maintain reproductive and behavioral isolation. The guenons, a

354 recent and diverse radiation that exhibit mixed species groups in which hybridization is rarely

355 observed, exemplify this phenomenon. By showing how species classification is dependent on

356 different aspects of face patterning and that this links with looking time toward con and

357 heterospecifics, our analyses support a role for guenon face patterns in species discrimination,

358 and identify specific face regions critical for this function. This parsing of critical signal

359 components is critical for understanding the phenotypic evolution of complex signals and

360 identifying relevant axes of signal variation for additional analyses.

$361 \quad$ Our occlude-reclassify analysis identified face regions critical to correct species

362 classification by a machine classifier in all guenon species included in our study. Critical regions

363 differed in both location and spread across the face, suggesting variation in potential use across

364 species. For some guenons, reliance on a single facial characteristic may be sufficient for species

365 discrimination. The best example of this in our data set is the putty nosed monkey, where our

366 machine classifier relied exclusively on the white nose spot to classify this species. That is, 
367 occlusion of any other region resulted in correct classification, but when the nose spot was

368 occluded classification failed. This result is reinforced by our experiments, in which putty nosed

369 monkey visual attention was driven wholly by the presence of nose spots. Putty nosed monkeys

370 exhibited a conspecific bias when presented with natural con- and heterospecific faces, as is

371 typical in primates, however including stimuli depicting heterospecifics with nose spots and

372 conspecifics without nose spots completely obscured this conspecific bias. This combination of

373 results illustrates the importance of nose spots in this species. It is worth noting that putty nosed

374 monkey nose spots are the most straightforward facial trait documented in our analysis (i.e. putty

375 nosed monkeys were only misclassified when the nose spot was occluded and occluding the nose

376 spot led to a high rate of misclassification) and the relative simplicity of the face and related

377 visual biases in this species is likely exceptional. On the whole, species discrimination signals in

378 a large radiation with varying patterns of sympatry are expected to be complex and

379 multidimensional, and it is likely that only some species can exhibit single-trait-based signals

380 and visual biases without the system breaking down. This is supported by our results showing

381 that for most guenon species our classifier relied on multiple face regions for species

382 discrimination.

Not all guenons exhibited critical face regions restricted to a single facial trait, and our

384 machine classifier sometimes relied on disparate face regions. In our data set, the mona monkey

385 is a good example of such a species. Like in putty nosed monkeys, our experiments with mona

386 monkeys supported these computational results. Mona monkeys exhibited a conspecific bias

387 across all trials, regardless of single trait manipulations, as well as an additional bias based on the

388 presence of eyebrow patches. Thus, eyebrow patches alone do not appear to be the sole focus of

389 attention in mona monkeys. We predict that additional manipulation of other face regions would 
390 be necessary to redirect their visual attention. Nonetheless, that mona monkey attention is still

391 influenced by this species-typical trait shows that it is important but not essential, a result

392 predicted by our computational analyses. It is unclear why mona monkeys would look longer at

393 stimuli without eyebrow patches, however it is possible that utilization of the whole face causes

394 increased attention to incongruency (e.g. conspecifics without eyebrow patches or

395 heterospecifics with them). Our results suggest that in mona monkeys, species discrimination

396 may be based on broader face information, and the perceptual processes involved in assessing

397 potential mates could be similar to generalized holistic face processing mechanisms observed in

398 other primates [47].

Our results suggest that guenons, while united by a general pattern of facial

400 diversification and the probable use of faces in mate choice, may vary across species in the

401 specific traits and processes that are involved in discriminating between conspecifics and

402 heterospecifics. Our pattern of results for putty nosed monkey nose spots and mona monkey

403 eyebrow patches is interesting because we know that both traits do contain sufficient information

404 to discriminate between species that share these features [24], yet they influence attention

405 differently in the two species. This disparity highlights the importance of testing receiver

406 perception directly. The fact that our experimental results with guenons line up with predictions

407 generated by our occlude-reclassify analysis implies that these computationally derived results

408 are biologically valid. Interestingly, we found no sex differences in visual biases for either

409 species, suggesting that selective pressures on species discrimination signaling and preference

410 traits are similar between sexes.

411 In guenons, an observed lack of hybrids in most polyspecific groups [21] is notable given

412 that hybridization is known to be possible between many guenon species [21,23,27], and 
413 indicates the existence of pre-mating barriers to reproduction. Increased eye gaze is associated

414 with increased mating interest in humans [48] and non-human primates [44,49], suggesting that

415 our experimental results would generalize to mating contexts in guenons. Combined with

416 previous work [10,23,24,26,27], our results support the hypothesis that guenon face patterns play

417 a role in mate choice and reproductive isolation in this group. However, it remains possible that

418 the selection pressure for species discrimination traits in guenons arises partially or entirely from

419 other functions where behavioral coordination or avoidance between species is advantageous,

420 such as in foraging decisions [20,23]. Careful field observations would be needed to distinguish

421 between such possibilities.

422 Our occlude-reclassify approach is a novel method for identifying the distribution of

423 information in complex signals and can be used for any question that can be conceptualized as a

424 discrimination problem and analyzed using machine classification. This method therefore has

425 broad utility within sensory ecology and could help to better understand the link between form

426 and function in the complex signals that are common in many animal groups. The objectivity of

427 the approach is important, as it allows researchers to intelligently target specific signal

428 components for further analysis without reference to their own perceptions of their salience. This

429 is particularly important when studying species with sensory and perceptual systems very

430 different from our own [50,51]. Where possible, combining this approach with a biologically

431 realistic classification scheme, such as classification within a perceptual face space based on

432 eigenface scores [36] as used here, increases the biological validity of results.

433 Our research broadens our understanding of how morphology and social decision-making

434 can interact to structure interactions between species living in sympatry. In guenons, facial

435 features like white nose spots are highly salient, attention-grabbing, and distinctive, and our 
combined results demonstrate the importance of these traits in species discrimination. Guenon

behavioral repertoires, such as nose-to-nose touching observed in wild putty nosed monkeys

438 (SW, personal observation) and red-tailed monkeys [52], further reflect the importance and

439 biological relevance of these traits. Primates preferentially attend to facial information $[53,54]$,

440 making face patterns particularly suited to influencing behavior and decision-making in con- and

441 heterospecifics. The evolution of signals facilitating species discrimination may be a major

442 driver of biological diversity, and our work linking mating signal form and function in a recent

443 and diverse primate radiation highlights how such evolutionary processes can be important in

444 generating animal phenotypes.

\section{$446 \quad$ References}

447 1. Coyne JA, Orr HA. 2004 Speciation. Sunderland, MA: Sinauer Associates, Inc.

448 2. Andersson MB. 1994 Sexual selection. Princeton, NJ: Princeton University Press.

449 3. Dawkins MS, Guilford T. 1997 Conspicuousness and diversity in animal signals. In

450 Communication (eds DH Owings, MD Beecher, NS Thompson), pp. 55-75. Boston, MA:

$451 \quad$ Springer US.

452 4. Brown WL, Wilson EO. 1956 Character displacement. Syst. Zool. 5, 49-64.

453 5. Pfennig KS, Pfennig DW. 2009 Character displacement: ecological and reproductive 454 responses to a common evolutionary problem. Q. Rev. Biol. 84, 253-276.

$455 \quad$ (doi:10.1086/605079)

456 6. Higgie M, Chenoweth S, Blows MW. 2000 Natural selection and the reinforcement of mate recognition. Science 290, 519-521. (doi:10.1126/science.290.5491.519)

7. Lukhtanov VA, Kandul NP, Plotkin JB, Dantchenko A V, Haig D, Pierce NE. 2005 Reinforcement of pre-zygotic isolation and karyotype evolution in Agrodiaetus butterflies. Nature 436, 385-9. (doi:10.1038/nature03704)

8. McNaught MK, Owens IPF. 2002 Interspecific variation in plumage colour among birds: species recognition or light environment? J. Evol. Biol. 15, 505-514.

9. Kameda Y, Kawakita A, Kato M. 2009 Reproductive Character Displacement in Genital Morphology in Satsuma Land Snails. Am. Nat. 173, 689-697. (doi:10.1086/597607)

10. Allen WL, Stevens M, Higham JP. 2014 Character displacement of Cercopithecini primate visual signals. Nat. Commun. 5, 4266. (doi:10.1038/ncomms5266)

11. Pfennig KS, Rice AM. 2014 Reinforcement generates reproductive isolation between neighbouring conspecific populations of spadefoot toads. Proc. R. Soc. B Biol. Sci. 281, 20140949-20140949. (doi:10.1098/rspb.2014.0949) 
12. Stanger-Hall KF, Lloyd JE. 2015 Flash signal evolution in Photinus fireflies: Character displacement and signal exploitation in a visual communication system. Evolution 69, 666682. (doi:10.1111/evo.12606)

13. Gordon NM, Ralph MZ, Stratman KD. 2017 Rapid character displacement of different call parameters in closely related treefrogs (Hyla cinerea and H. gratiosa). Behav. Ecol. Sociobiol. 71. (doi:10.1007/s00265-017-2341-1)

14. Mendelson TC, Shaw KL. 2012 The (mis) concept of species recognition. Trends Ecol. Evol. 27, 421-427. (doi:10.1016/j.tree.2012.04.001)

15. Mallet J. 2005 Hybridization as an invasion of the genome. Trends Ecol. Evol. 20, 229-237. (doi:10.1016/j.tree.2005.02.010)

16. Groves CP. 2005 Order Primates. In Mammal Species of the World: A Taxonomic and Geographic Reference, pp. 111-184. Baltimore, MD: Johns Hopkins University Press.

17. Grubb P, Butynski TM, Oates JF, Bearder SK, Disotell TR, Groves CP, Struhsaker TT. 2003 Assessment of the Diversity of African Primates. Int. J. Primatol. 24, 1301-1357. (doi:10.1023/B:IJOP.0000005994.86792.b9)

18. Hart JA et al. 2012 Lesula: a new species of Cercopithecus monkey endemic to the Democratic Republic of Congo and implications for conservation of Congo's central basin. PloS One 7, e44271. (doi:10.1371/journal.pone.0044271)

19. Tosi AJ, Detwiler KM, Disotell TR. 2005 X-chromosomal window into the evolutionary history of the guenons (Primates: Cercopithecini). Mol. Phylogenet. Evol. 36, 58-66. (doi:10.1016/j.ympev.2005.01.009)

20. Jaffe KE, Isbell LA. 2011 The guenons: polyspecific associations in socioecological perspective. In Primates in Perspective (eds CJ Campbell, A Fuentes, KC MacKinnon, SK Bearder, RM Stumpf), pp. 277-300. New York, NY: Oxford University Press.

21. Detwiler KM, Burrell AS, Jolly C. 2005 Conservation implications of hybridization in African cercopithecine monkeys. Int. J. Primatol.

22. Cords M. 2012 The behavior, ecology, and social evolution of Cercopithecine monkeys. In The Evolution of Primate Societies (eds JC Mitani, RA Palombit, JB Silk), pp. 91-112. Chicago: University of Chicago Press.

23. Kingdon J. 1997 The Kingdon guide to African mammals. London: Academic Press.

24. Allen WL, Higham JP. 2015 Assessing the potential information content of multicomponent visual signals: a machine learning approach. Proc. R. Soc. B Biol. Sci. (doi:10.1098/rspb.2014.2284)

25. Bradley BJ, Mundy NI. 2008 The primate palette: The evolution of primate coloration. Evol. Anthropol. 17, 97-111. (doi:10.1002/evan.20164)

26. Kingdon J. 1988 What are face patterns and do they contribute to reproductive isolation in guenons. In A Primate Radiation: Evolutionary Biology of the African Guenons, pp. 227245.

27. Kingdon J. 1980 The role of visual signals and face patterns in African forest monkeys (guenons) of the genus Cercopithecus. Trans. Zool. Soc. Lond. 35, 425-475.

(doi:10.1111/j.1096-3642.1980.tb00062.x)

28. Pfennig DW, Pfennig KS. 2010 Character Displacement and the Origins of Diversity. Am. Nat. 176, S26-S44. (doi:10.1086/657056)

29. Pfennig KS. 2016 Reinforcement as an initiator of population divergence and speciation. Curr. Zool. 62, 145-154. (doi:10.1093/cz/zow033) 
30. Stuart YE, Inkpen SA, Hopkins R, Bolnick DI. 2017 Character displacement is a pattern: so, what causes it? Biol. J. Linn. Soc. 121, 711-715. (doi:10.1093/biolinnean/blx013)

31. Patricelli GL, Hebets EA. 2016 New dimensions in animal communication: the case for complexity. Curr. Opin. Behav. Sci. 12, 80-89. (doi:10.1016/j.cobeha.2016.09.011)

32. Turk M, Pentland A. 1991 Eigenfaces for Recognition. J. Cogn. Neurosci. 3, 71-86. (doi:10.1162/jocn.1991.3.1.71)

33. Perera TA, Collins J. 2015 Imaged based species recognition system. In 9th International Conference on Sensing Technology (ICST), pp. 195-199. IEEE.

34. Shiau Y-H, Lin S-I, Chen Y-H, Lo S-W, Chen C-C. 2012 Fish observation, detection, recognition and verification in the real world. In Proceedings of the International Conference on Image Processing, Computer Vision, and Pattern Recognition (IPCV), pp. 16. The Steering Committee of The World Congress in Computer Science, Computer Engineering and Applied Computing (WorldComp).

35. Weeks P, O’Neill M, Gaston K, Gauld I. 1999 Species-identification of wasps using principal component associative memories. Image Vis. Comput. 17, 861-866. (doi:10.1016/S0262-8856(98)00161-9)

36. Chang L, Tsao DY. 2017 The code for facial identity in the primate brain. Cell 169, 10131028.e14. (doi:10.1016/j.cell.2017.05.011)

37. Winters S, Dubuc C, Higham JP. 2015 Perspectives: the looking time experimental paradigm in studies of animal visual perception and cognition. Ethology 121, 625-640. (doi:10.1111/eth.12378)

38. Fujita K. 1987 Species recognition by five macaque monkeys. Primates 28, 353-366. (doi:10.1007/BF02381018)

39. Demaria C, Thierry B. 1988 Responses to animal stimulus photographs in stumptailed macaques (Macaca arctoides). Primates 29, 237-244. (doi:10.1007/BF02381125)

40. Dufour V, Pascalis O, Petit O. 2006 Face processing limitation to own species in primates: a comparative study in brown capuchins, Tonkean macaques and humans. Behav. Processes 73, 107-13. (doi:10.1016/j.beproc.2006.04.006)

41. Méary D, Li Z, Li W, Guo K, Pascalis O. 2014 Seeing two faces together: preference formation in humans and rhesus macaques. Anim. Cogn. 17, 1107-1119.

(doi:10.1007/s10071-014-0742-3)

42. Rakotonirina H, Kappeler PM, Fichtel C. 2018 The role of facial pattern variation for species recognition in red-fronted lemurs (Eulemur rufifrons). BMC Evol. Biol. 18.

(doi:10.1186/s 12862-018-1126-0)

43. Zeiler MD, Fergus R. 2014 Visualizing and Understanding Convolutional Networks. In Computer Vision - ECCV 2014 (eds D Fleet, T Pajdla, B Schiele, T Tuytelaars), pp. 818833. Cham: Springer International Publishing. (doi:10.1007/978-3-319-10590-1_53)

44. Dubuc C, Allen WL, Cascio J, Lee DS, Maestripieri D, Petersdorf M, Winters S, Higham JP. 2016 Who cares? experimental attention biases provide new insights into a mammalian sexual signal. Behav. Ecol. 27, 68-74. (doi:10.1093/beheco/arv117)

45. Bates D, Mächler M, Bolker B, Walker S. 2015 Fitting linear mixed-effects models using lme4. J. Stat. Softw. 67, 1-48. (doi:10.18637/jss.v067.i01)

46. R Core Team. 2018 R: a language and environment for statistical computing, v3.5.2. R Foundation for Statistical Computing, Vienna, Austria. See https://www.R-project.org.

47. Dahl CD, Wallraven C, Bülthoff HH, Logothetis NK. 2009 Humans and macaques employ similar face-processing strategies. Curr. Biol. 19, 509-13. (doi:10.1016/j.cub.2009.01.061) 
48. Shimojo S, Simion C, Shimojo E, Scheier C. 2003 Gaze bias both reflects and influences preference. Nat. Neurosci. 6, 1317-1322. (doi:10.1038/nn1150)

49. Dubuc C, Allen WL, Maestripieri D, Higham JP. 2014 Is male rhesus macaque red color ornamentation attractive to females? Behav. Ecol. Sociobiol. 68, 1215-1224. (doi:10.1007/s00265-014-1732-9)

50. Endler JA. 1990 On the measurement and classification of colour in studies of animal colour patterns. Biol. J. Linn. Soc. 41, 315-352.

51. Bennett ATD, Cuthill IC, Norris K. 1994 Sexual selection and the mismeasure of color. Am. Nat. , 848-860.

52. Estes R. 1991 The Behavior Guide to African Mammals: Including Hoofed Mammals, Carnivores, Primates. Berkeley \& Los Angeles, CA: University of California Press.

53. Tsao DY, Schweers N, Moeller S, Freiwald WA. 2008 Patches of face-selective cortex in the macaque frontal lobe. Nat. Neurosci. 11, 877-879. (doi:10.1038/nn.2158)

54. Kano F, Tomonaga M. 2009 How chimpanzees look at pictures: a comparative eye-tracking study. Proc. R. Soc. B Biol. Sci. 276, 1949-1955. (doi:10.1098/rspb.2008.1811)

\section{Acknowledgements}

We thank Allegra Depasquale and Laura Newman for assistance coding looking time videos, and Kathryn Yee for assistance with stimuli preparation. Special thanks the directors and staff of CERCOPAN sanctuary, particularly Claire Coulson and Isabelle Theyse, for access to the facility and support during data collection.

\section{Data accessibility}

The guenon face image database, experimental looking time data, and all project code will be uploaded to Dryad. During review, all files are available at: http:/www.dropbox.com/sh/1yf97kbhggzi5a8/AAAJ0dQMEqeFOMf9aHzGBMHNa?dl=0 


\section{$589 \quad$ Figure 1}

590 Example experimental stimulus pairs. Subjects were shown a pair of stimulus images consisting

591 of a conspecific and a heterospecific. Facial traits (nose spots for putty nosed monkeys and

592 eyebrow patches for mona monkeys) were varied across trials, with conspecifics paired with a

593 heterospecific species that shares the facial trait (row 1) and one that does not (rows 2 and 3 ).

594 Conspecifics were displayed either naturally (rows 1 and 2) or with the facial trait removed (row

595 3). All subjects participated in all three trial types. Trial order and stimulus image side were

596 counterbalanced across subjects.

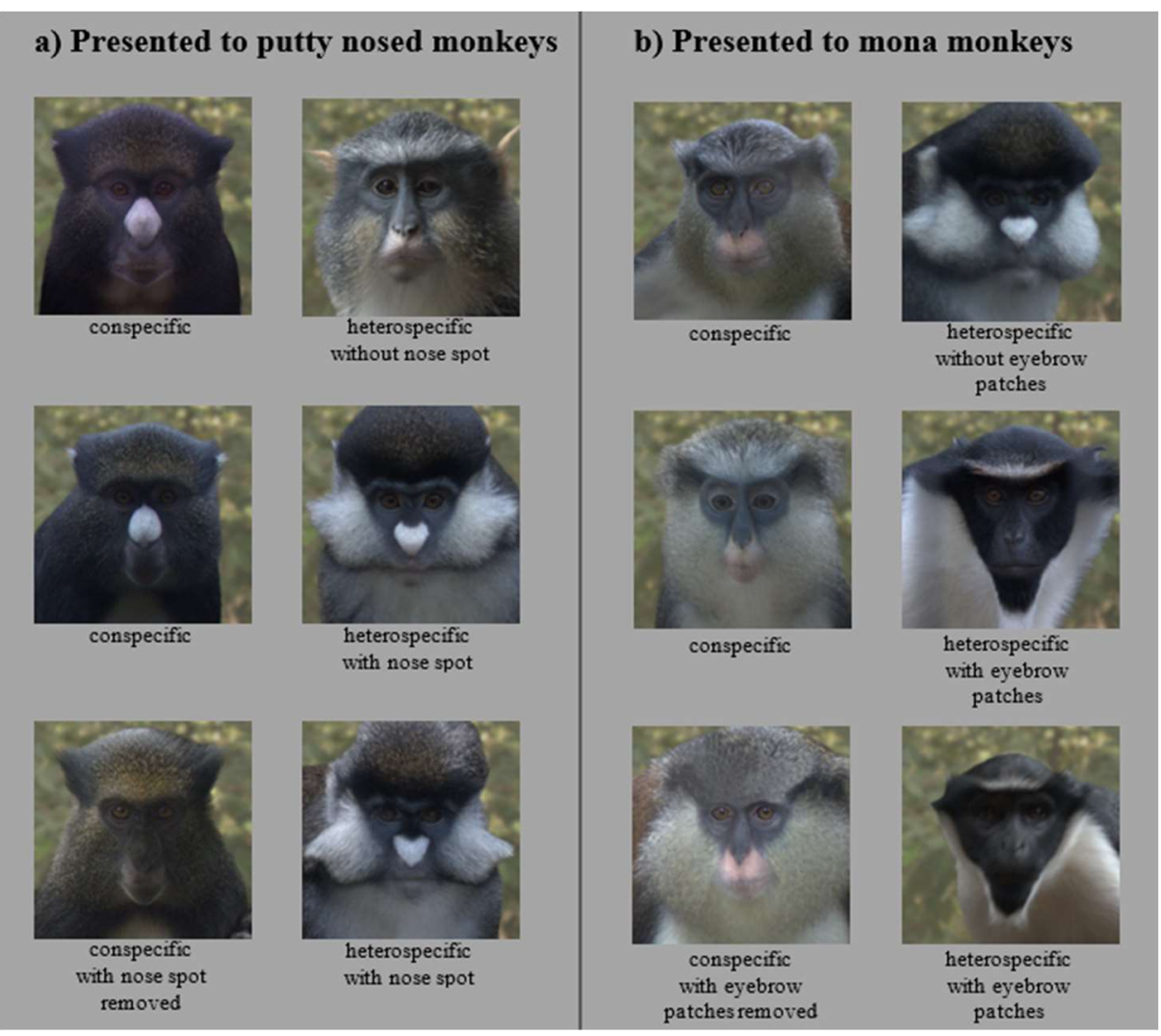




\section{$599 \quad$ Figure 2}

600 Average guenon face with an occluder shown in the top left. The occluder is depicted in black

601 for maximal visibility, but in analyses presented here is set to the mean face color of the relevant

602 species. During the occlude-reclassify analysis, the occluder is slid across the image and the

603 image re-classified; an incorrect classification at a given occluder location indicates the presence

604 of face information critical to correct classification. Analyses are run on hemi-faces to account

605 for facial symmetry. Image borders outside the radius of the occluder are not tested; the dashed

606 line encloses the region of the image analyzed using the occlude-reclassify procedure.

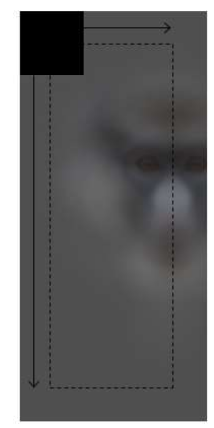

608 


\section{Figure 3}

611 Likelihood of correct classification based on occlusion of different face regions. Species average faces are displayed on the left and

612 heatmaps identifying critical face regions on the right. Sample size is reported as $n=$ number of individuals (number of total images).
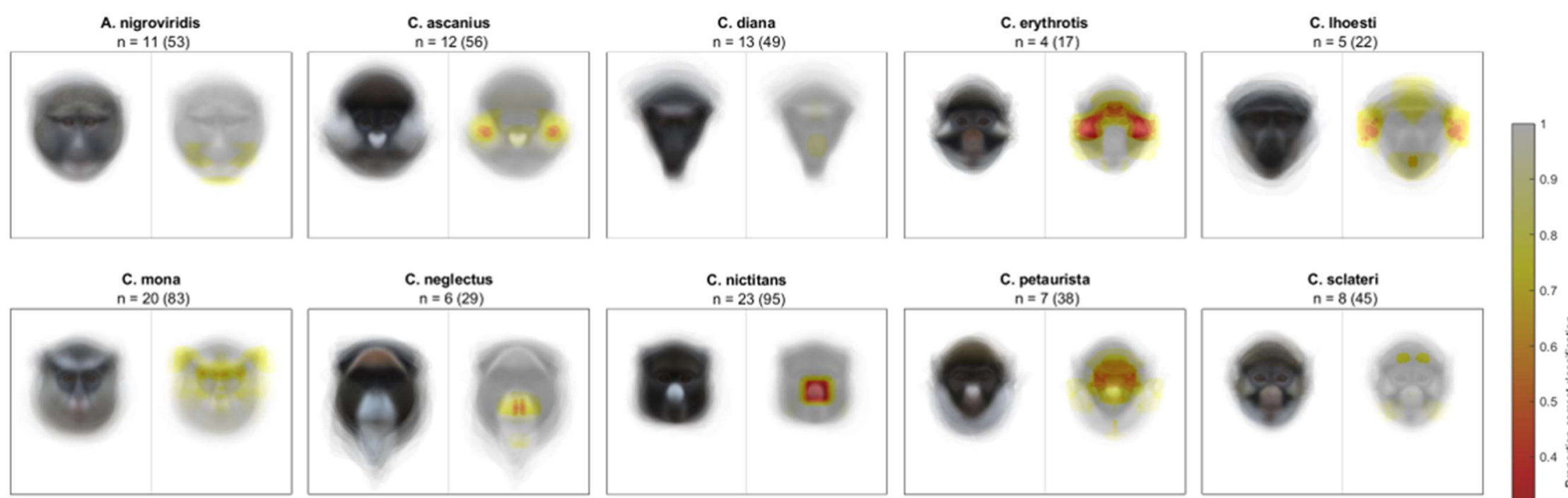

613
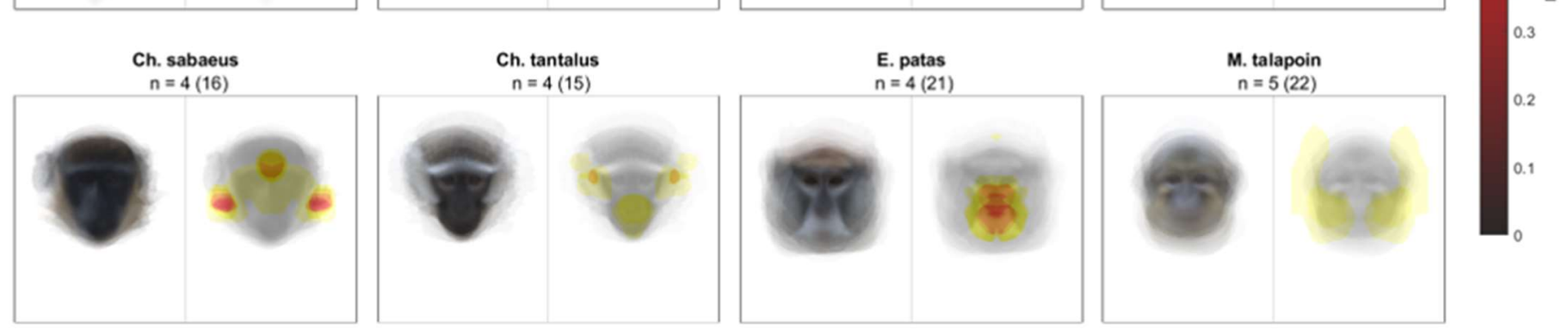

614 


\section{$615 \quad$ Figure 4}

616 Variation across species in face regions identified as essential for correct species classification.

617 The proportion of the face misclassified (y-axis) indicates the spread of essential regions across

618 the face; higher values signify broader spread and lower values more concentrated regions. The

619 mean classification error (x-axis) measures the relative importance of identified features; higher

620 values indicate higher rates of misclassification, suggesting identified regions are particularly

621 essential for correct species classification. Experimental results are presented for C. mona and C.

622 nictitans (Figure 5).

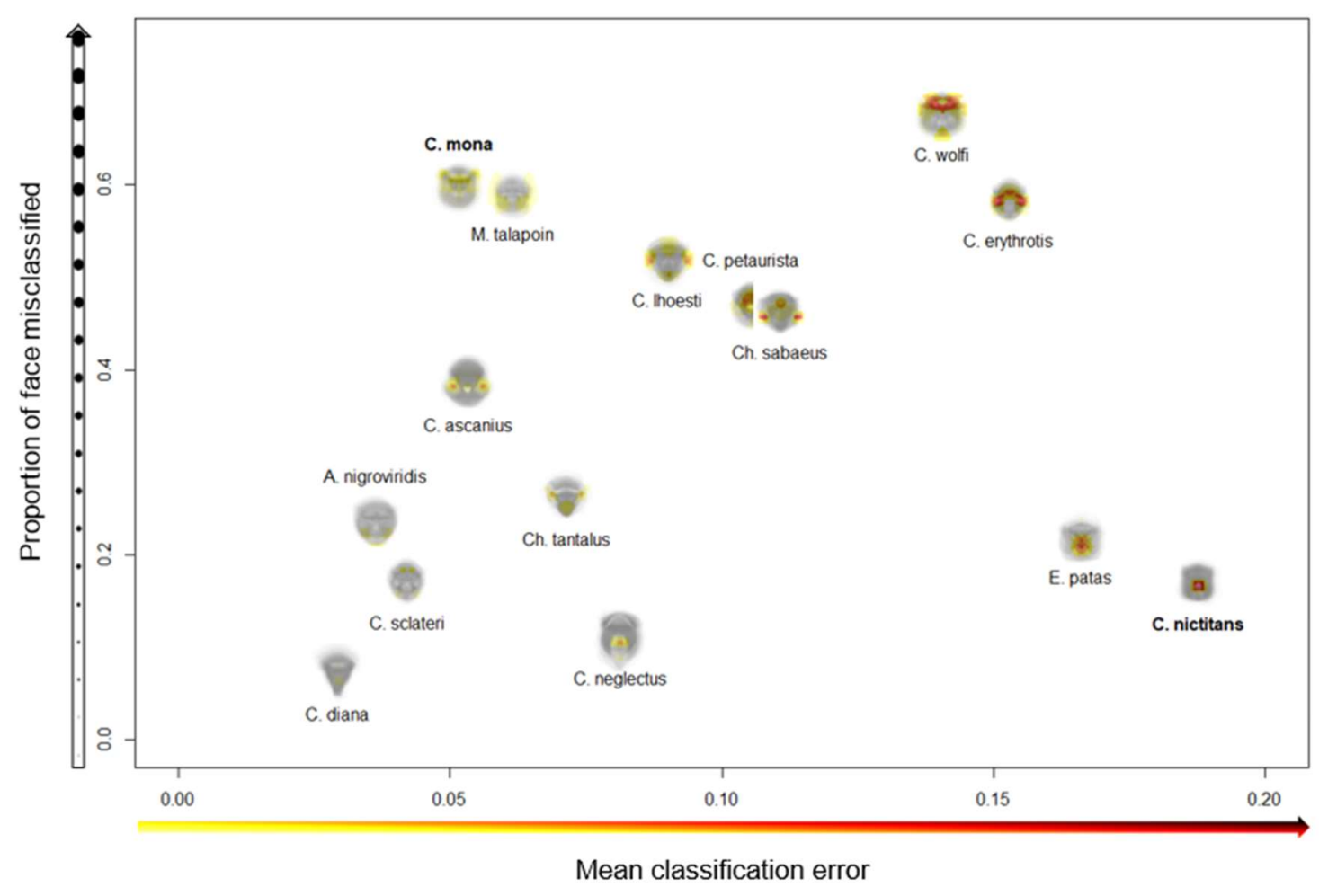




\section{Figure 5}

626 Species and trait biases observed during looking time tasks with (a) putty nosed monkeys and (b)

627 mona monkeys. Leftmost plots depict differences in looking time in trials consisting of

628 conspecifics and heterospecifics without the relevant facial trait. Center and right plots depict

629 looking time differences across all trials - which also include heterospecifics with the relevant

630 facial trait and conspecifics without it - with species biases depicted in the center and trait biases

631 on the right. Results are based on 18 putty nosed monkeys and 16 mona monkeys. Each subject

632 participated in three trials (see Figure 1 for example stimuli for each trial type). Error bars

633 indicate the standard error of the mean.

a) Putty nosed monkeys
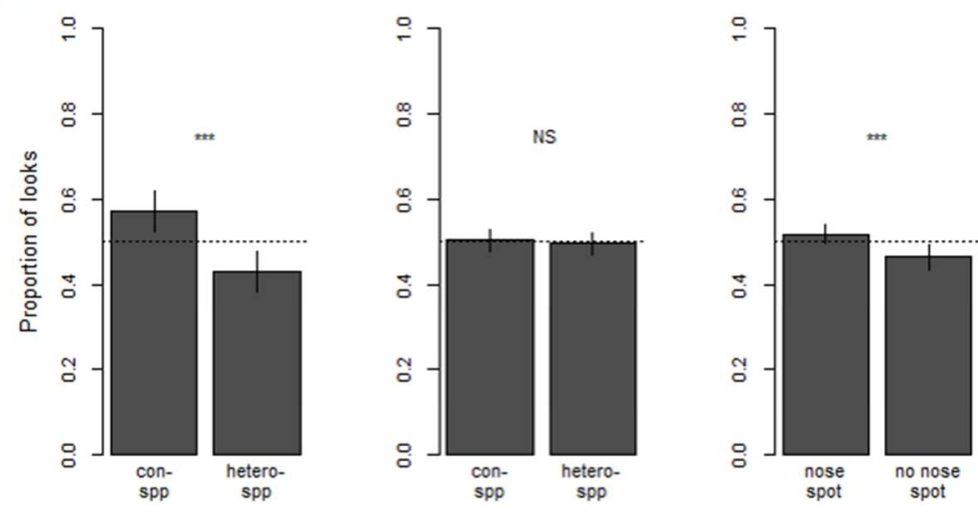

b) Mona monkeys
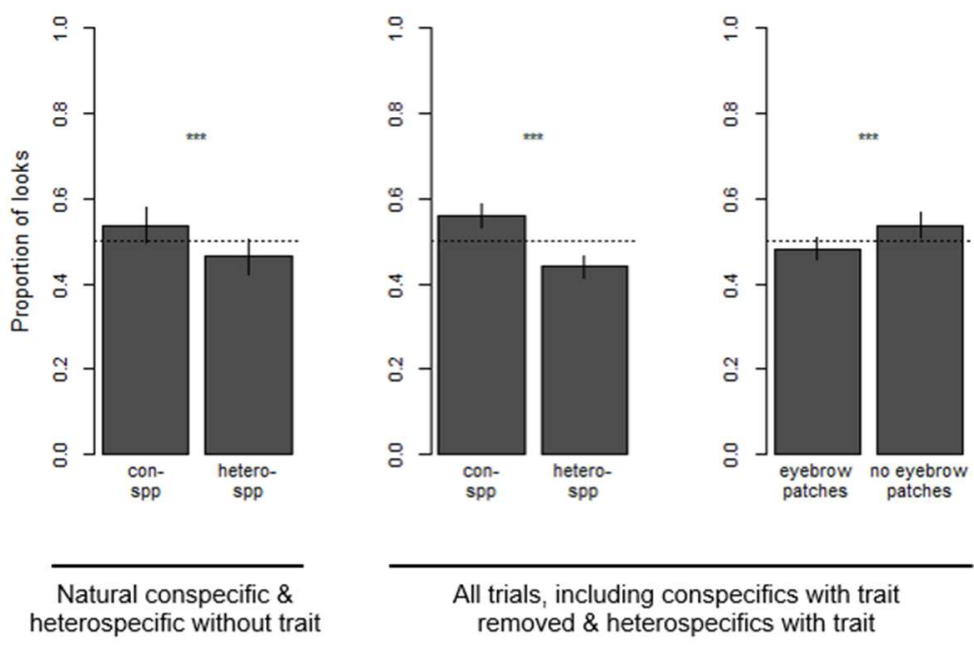